\title{
Improving Time-Frequency Domain Sleep EEG Classification via Singular Spectrum Analysis
}

\author{
Sara Mahvash Mohammadi ${ }^{\mathrm{a}, *}$, Samaneh Kouchaki ${ }^{\mathrm{b}},{\text { Mohammad } \text { Ghavami }^{\mathrm{a}} \text {, Saeid Sanei }}^{\mathrm{b}}$ \\ ${ }^{a}$ Department of Engineering and Design, London South Bank University,London, UK \\ ${ }^{b}$ Faculty of Engineering and Physical Sciences, University of Surrey, Guildford, UK
}

\begin{abstract}
Background: Manual sleep scoring is deemed to be tedious and time consuming. Even among automatic methods such as Time-Frequency (T-F) representations, there is still room for more improvement.

New method: To optimise the efficiency of T-F domain analysis of sleep electroencephalography (EEG) a novel approach for automatically identifying the brain waves, sleep spindles, and K-complexes from the sleep EEG signals is proposed. The proposed method is based on singular spectrum analysis (SSA). The single-channel EEG signal (C3-A2) is initially decomposed and then the desired components are automatically separated. In addition, the noise is removed to enhance the discrimination ability of features. The obtained T-F features after preprocessing stage are classified using a multi-class support vector machines (SVM) and used for the identification of four sleep stages over three sleep types. Furthermore, to emphasize on the usefulness of the proposed method the automatically-determined spindles are parameterised to discriminate three sleep types.

Result: The four sleep stages are classified through SVM twice: with and without preprocessing stage. The mean accuracy, sensitivity, and specificity for before the preprocessing stage are: $71.5 \pm 0.11 \%, 56.1 \pm 0.09 \%$ and $86.8 \pm 0.04 \%$ respectively. However, these values increase significantly to $83.6 \pm 0.07 \%, 70.6 \pm 0.14 \%$ and $90.8 \pm 0.03 \%$ after applying SSA.

Comparison with existing method: The new T-F representation has been compared with the existing benchmarks. Our results prove that, the proposed method well outperforms the previous methods in terms of identification and representation of sleep stages.

Conclusion: Experimental results confirm the performance improvement in terms of classification rate and also representative T-F domain.
\end{abstract}

Keywords: Electroencephalogram, Feature extraction, Sleep, Singular spectrum analysis, Time-Frequency representation

\section{Introduction}

Sleep research has applications in medical science, psychology, and bioengineering. Amongst different sleep disorders such as sleep apnoea, insomnia and narcolepsy, many 5 of them divulge themselves through sleep disturbances like depression and schizophrenia [1]. Sanitising and studying sleep can be accomplished through polysomnographic ${ }_{25}$ (PSG) measurements, encompassing EEG, electromyogram (EMG), and electrooculogram (EOG) [2]. The Rechtschaffen and Kales standard $(\mathrm{R} \& \mathrm{~K})[3]$ and American Academy of Sleep Medicine (AASM) [4] are commonly used to guideline, regulate, and govern the standards for classifying and monitoring the sleep stages. R\&K described the sleep as a six sequence stages including: awake, stage 1 , stage 2 , 15 stage 3 , stage 4 , and rapid eye movement (REM). Stages $1,2,3$, and 4 are categorised under non-rapid eye movement (NREM). However, sleep stages 3 and 4 are recently

\footnotetext{
* Corresponding author
}

grouped into one stage by AASM and assigned N3 stage. Thus, NREM sleep is divided into three stages: N1, N2, and N3.

Generally speaking, awake stage is observed at the start of the sleep and is known as a shift stage from complete awareness to a half-sleepy condition. This stage is characterised mainly by its frequency range of 8 to $12 \mathrm{~Hz}$ that contains alpha rhythms, eye movements, and high muscle tone. Stage N1 is referred to as a moving stage from wakefulness to sleep. This stage entails a low-voltage, mixed frequency EEG tracing accompanied by high amplitude theta waves. It is as short as 5-10 minutes. Stage N2 is known as sleep baseline and lasts for approximately 20 minutes. This stage can be identified by the incidence of sleep spindles and K-complexes. Sleep spindles are bursts of rapid rhythmic brain wave activity which appear within the frequency range of $12-14 \mathrm{~Hz}$ and last for approximately 0.5 second. K-complex is an abrupt peak in time that spreads in frequency domain [5].

Stage N3 is characterized by the presence of slow wave 


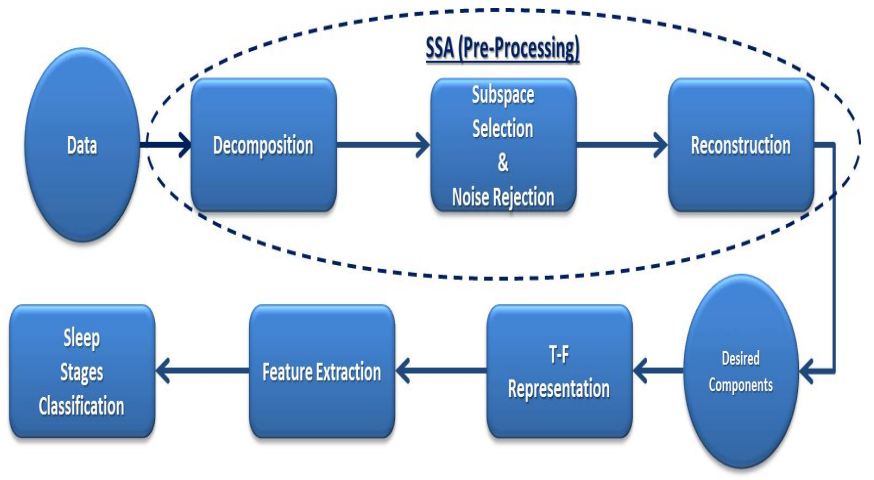

Figure 1: Block diagram of the proposed sleep scoring approach.

activity (SWA) with frequencies up to $2 \mathrm{~Hz}$ and amplitude of more than $75 \mu \mathrm{V}$. At this stage spindles may be gen- ${ }^{95}$ 40 erated. However, the amount of sleep spindles decrease as the sleep deepens. $17-20 \%$ of the total sleep time is in stage N3. This stage is also called as slow wave sleep (SWS) Following the NREM stages, REM sleep stage corresponds to dreaming and contributes to $20-25 \%$ of $\mathrm{a}^{100}$ 45 normal sleep. It is defined as an occurrence of rapid eye movement under closed eyelids [6].

Study of sleep stages has gained appreciation from the researchers. This is because of the fact that different sleep disorders and sleep deprivation impact on individuals as ${ }^{105}$ 50 well as public health to the large extend [7]. Manual sleep stage classification and scoring is performed by experts and clinicians. However, this method is subject to human error. Moreover, the manual method is a very tedious and arduous exercise which leads to a low reliabil- ${ }^{110}$

${ }_{55}$ ity and high subjective error [8]. In order to effectively tackle this problem different automatic classification of sleep stages based on multi-channel EEG signals [9][10] or single-channel EEG signal [11][12] are highly desired.

Automatic sleep detection is an active area in research. ${ }^{115}$

60 Nonetheless, T-F methods compared with other techniques have received more attention due to existing clear T-F patterns in sleep EEG. Most of the solutions for sleep EEG analysis like Fourier transform are only capable of providing generic frequency specification while the transient ${ }^{120}$ 65 events are not discussed explicitly. Wavelet transform (WT) is another technique for sleep analysis. However, there are a couple of drawbacks associated with WT including dependency of its results to the choice of mother wavelet and also the fact that the wavelet basis functions ${ }^{125}$

70 are not data dependent. In addition, unlike Fourier transform which only exploits sinusoid functions or in case of wavelet transform which uses mother wavelet, matching pursuit(MP) benefits from a large dictionary size which gives high flexibility in signal structure identification and ${ }^{130}$ 75 parameterizations and better deals with nonstationarity of the signals. [13].

For years, different T-F representation methods have been used for automatic sleep stage classification [12, 14, 15]. However, these methods are impacted by interferences ${ }^{135}$ so stemmed from unwanted components. Durka in [16] proposed automatic detection and parametrization of sleep events on the premise of MP spectrum. However, in stage N3 the alpha wave totally vanishes and non-frequent low amplitude spindles may occur. The spindles have frequency within alpha range but usually occur in the absence of normal brain alpha wave. In addition, the key components for classifying the sleep stages are brain waves, sleep spindles, and K-complexes. In the T-F energy map for various sleep stages in [16] all components including desired and undesired are plotted. Hence the purpose of this paper is to append a preprocessing stage to address these issues.

Singular spectrum analysis (SSA) method is leveraged for automatic identification and extraction of desired components: brain waves, spindles and K-Complex in their actual locations. SSA has had emerging application in trend extraction, time series decomposition, periodicity extraction, signal extraction, noise reduction, and filtering $[17,18]$. In time series analysis, SSA plays a major role as a robust technique for tackling a diverge range of issues in practice. Recently, in terms of biomedical signal processing application, SSA has been used for restoration of heart sound from lung sound [19], and separating ECG and EMG [20]. It has been also considered for estimation of detailed gait analysis and parameters from a wearable devices [21]. More recently, SSA has been employed in signal processing applications such as processing of multichannel EEG signals for classification of five sleep stages [9] and evaluation of alpha and delta waves for more accurate determination of the transition between two sleep stages [22].

This paper describes the extraction of desired components from EEG signals prior to applying T-F transform and classification. The overall strategy of this work is illustrated in Figure 1. Note that the EEG sleep signals are deemed to have nonstationarity. SSA benefits from the elements of classical time series analysis, linear algebra, multivariate geometry, multivariate statistics, dynamical systems, and signal processing, and can exploit the signal nonsstationarity [17]. In addition, the noise component can be removed during the SSA decomposition. This is envisaged to be another significant merit of SSA as a preprocessing stage.

Here, a new constrained SSA has been proposed. Then, the extracted features from both methods i.e. with and without preprocessing stage, were classified with the aid of the support vector machine (SVM) classifier. Classification accuracy of awake, N1+ REM, N2, and SWS is improved through using SSA preprocessing.

In this work, SSA is applied to sleep EEG analysis. In another study, pulse oximetry and heart rate sensors are employed in order to diagnose sleep disorders [23]. Therefore, as a future work, the current application can be improved by incorporating the joint motion [21], heart rate, and EEG analysis of human sleep. 
Sleep EEG in the course of NREM is characterised with sleep spindles. The degree of hyperpolarization of thalamocortical cells (TC) justifies the given fluctuations and the causing method. With the aid of fast Fourier transform (FFT), spectral analysis of the NREM displays frequency specific modulation of spindle frequency activity which varies based on the homeostatic sleep pressure [24][25]. Hence as another SSA application, through pawe also focus on the impact of different sleep types on the extracted spindles characteristics. In other words, the effect of enhanced sleep pressure after sleep deprivation (SD) and low sleep pressure after sleep extension (SE) on spindle characteristics is analysed using mean amplitude, density (i.e. the number of sleep spindles per 20 seconds epoch), duration, and frequency. After that, the spindles' features are employed as an input to the SVM classifier to classify normal sleep (SN), SE, and SD.

The rest of this paper is organised as follows: section 2 overviews the fundamentals of the employed method. Section 3 reveals the experimental results obtained and and discuss the results. Finally, the last section draws the concluding points.

\section{Materials and Methods}

\subsection{Matching Pursuit}

MP has been introduced by Mallat and Zhang in [26]. MP application is based on adaptive delineation of signal (y) with functions selected from a wide collection of waveforms, known as dictionary $(\mathbf{F})$. In the initial stage, the waveform that best fits the signal $\left(\mathbf{f}_{\gamma 0}\right)$ is selected from the dictionary $\mathbf{F}$. Then, in each iteration $n$ we have [16]:

$$
\left\{\begin{array}{l}
R^{0} \mathbf{y}=\mathbf{y} \quad \mathbf{f} \in \mathbf{H} \\
R^{n} \mathbf{y}=\left\langle R^{n} \mathbf{y}, \mathbf{f}_{\gamma n}\right\rangle \mathbf{f}_{\gamma n}+R^{n+1} \mathbf{y} \\
\mathbf{f}_{\gamma n}=\arg \max _{\mathbf{f}_{\gamma i}} \mathbf{f}_{\gamma i} \in \mathbf{F}\left|\left\langle R^{n} \mathbf{y}, \mathbf{f}_{\gamma i}\right\rangle\right|
\end{array}\right.
$$

Let $\mathbf{H}$ be the Hilbert space. $\mathbf{f}_{\gamma n}$ and $R^{n} \mathbf{y}$ are the waveform matched to the signal and the remaining signal after each iteration respectively and $\left\langle R^{n} \mathbf{y}, \mathbf{f}_{\gamma n}\right\rangle$ refers to crosscorrelation. The perfect signal approximation is achieved in an infinite number of iterations. Nonetheless, practically, a few number of waveforms (after finite number of iterations) result in a good signal approximation.

$$
\mathbf{y}=\sum_{n=0}^{M}\left\langle R^{n} \mathbf{y}, \mathbf{f}_{\gamma n}\right\rangle \mathbf{f}_{\gamma n}=\sum_{n=0}^{M} a_{n} \mathbf{f}_{\gamma n}
$$

where $M$ is the total number of iterations. Functions $\mathbf{f}_{\gamma}$ are selected from dictionary of the Gabor functions. Gabor is the best filtering in the T-F domain and can provide the optimal T-F localization using the complete Dirac and Fourier bases [16]. Real valued continuous time Gabor functions are shown as:

$$
\mathbf{f}_{\gamma}(t)=N(\gamma) e^{-\pi\left(\frac{t-u}{s}\right)^{2}} \cos (\omega(t-u)+\varphi)
$$

where $N(\gamma)$ is a normalising factor of $\mathbf{f}_{\gamma}$ and $\lambda=\{u, \omega, s\}$ corresponds to the parameter of the Gabor function (translating, modulating, and scaling). T-F distribution of the energy of the signal can be driven from expansion (2). Hence, the Wigner distributions $W$ of the chosen function are added while the cross-terms are removed so that $[26][16]$ :

$$
e_{\mathbf{y}}(t, \omega)=\sum_{n=0}^{M}\left|\left\langle R^{n} \mathbf{y}, \mathbf{f}_{\gamma n}\right\rangle\right|^{2} W_{\mathbf{f}_{\gamma n}}(t, \omega)=\sum_{n=0}^{M} a_{n}^{2} W_{\mathbf{f} \gamma n}
$$

where $e_{\mathbf{y}}$ is the T-F distribution.

\subsection{Singular Spectrum Analysis}

SSA works based on how well the diverged components can be taken apart from each other [20]. The fundamental SSA approach entails two stages which complement each other; decomposition and reconstruction. Each of these two stages, in turn, encompasses two distinct stages. The first stage involves embedding accompanied by singular value decomposition (SVD) to decompose the signal. Stage two consisting of grouping and diagonal averaging, reconstruct the signal while exploiting it for further analysis .

\subsubsection{Decomposition}

In the case of basic univariate SSA, a time series $\mathbf{f}$ should be mapped into a matrix known as trajectory matrix:

$$
\begin{aligned}
\mathbf{X} & =\left[\mathbf{x}_{1}, \ldots, \mathbf{x}_{n}\right]=\left(x_{i j}\right)_{i, j=1}^{l, n} \\
& =\left(\begin{array}{ccccc}
f_{1} & f_{2} & f_{3} & \ldots & f_{n} \\
f_{2} & f_{3} & f_{4} & \ldots & f_{n+1} \\
\vdots & \vdots & \ddots & \vdots & \\
f_{l} & f_{l+1} & f_{l+2} & \ldots & f_{s}
\end{array}\right)
\end{aligned}
$$

with one-dimensional vectors $\mathbf{x}_{i}=\left[f_{i}, f_{i-2}, \ldots, f_{i+l-1}\right]^{T}$, where $n=s-l+1$ and $l$ is the window length $(1<l<s)$. Note that, the window length $l$ should be adequately large to cater the information about the data variation. It is evident from (5) that the trajectory matrix is a Hankel matrix where the diagonal elements $(i+j=$ const $)$ are equal $[17,27]$.

Following the previous stage, SVD is performed to decompose the trajectory matrix into its eigen subspaces. To this end, consider the covariance matrix $\mathbf{C}_{\mathbf{x}}=\mathbf{X X}^{T}$ with eigenvalues $\lambda_{1}, \lambda_{2}, \ldots, \lambda_{l}$ in the decreasing order $\left(\lambda_{1}>\lambda_{2}>\ldots>\lambda_{l}\right)$ and $q_{1}, q_{2}, \ldots, q_{l}$ corresponding eigenvectors; therefore, SVD of the trajectory matrix can be rewritten as:

$$
\mathbf{X}=\mathbf{X}_{1}+\mathbf{X}_{2}+\ldots+\mathbf{X}_{r}
$$

where $\mathbf{v}_{j}=\mathbf{X}^{T} \mathbf{q}_{j} / \sqrt{\lambda_{j}}, \mathbf{X}_{j}=\sqrt{\lambda_{j}} \mathbf{q}_{j} \mathbf{v}_{j}^{T}$, and

$$
r=\max \left(j ; \text { such that } \lambda_{j}>0\right)
$$


175 The set $\left(\lambda_{j}, \mathbf{q}_{j}, \mathbf{v}_{j}\right)$ is named the $j$-th eigentriple of the matrix $\mathbf{X}$. The definition of $\mathbf{X}_{j}$ is equivalent to the elementary matrix. Projecting a time series onto each eigenvector yields the corresponding temporal principal component (PC) [19, 28].

\subsubsection{Reconstruction}

In the initial step of reconstruction stage, the elementary matrices $\mathbf{X}_{j}$ are splitted into several groups and then the matrices within each group are summed [17]. Therefore, each group is displayed by the related matrix $\tilde{\mathbf{X}}_{g} \subset{ }^{190}$ $\mathbb{R}^{l \times n}$ where:

$$
\mathbf{X}=\sum_{g=1}^{g_{t}} \tilde{\mathbf{X}}_{g}
$$

in which, $\tilde{\mathbf{X}}_{g}$ represents the sum of the elementary matrices ${ }^{195}$ within the group $g, g_{t}$ specifies the total number of groups, and index $g$ refers to the $g$-th subgroup of eigentriples. After completing the split stage, a specific $\left(\tilde{\mathbf{X}}_{g}\right)$ is chosen and then Hankelization procedure (averaging along entries with indices $i+j=$ const) reconstructs the subseries. $\operatorname{In}_{200}$ this case if $\tilde{x}_{i j}$ points to an element of the matrix $\left(\tilde{\mathbf{X}}_{g}\right)$, $k$-th term of the new reconstructed series $\tilde{x}_{i j}$ is computed by making the average of all along all $i, j$ in a way that $(i+j=k+1)$. Therefore, the following parameters can be initialized as $k=1, \tilde{f}_{1}=\tilde{x}_{11}$ and for $k=2, \tilde{f}_{2}=$ $\left(\tilde{x}_{12}+\tilde{x}_{21}\right) / 2$ and so on $[17]$.

$$
\begin{aligned}
\tilde{\mathbf{X}}_{g} & =\left(\begin{array}{cccc}
\tilde{x}_{11} & \tilde{x}_{12} & \ldots & \tilde{x}_{1, n} \\
\tilde{x}_{21} & \tilde{x}_{22} & \ldots & \tilde{x}_{2, n+1} \\
\vdots & \vdots & \ddots & \vdots \\
\tilde{x}_{l, 1} & \tilde{x}_{l, l+1} & \ldots & \tilde{x}_{l, s}
\end{array}\right) \\
\tilde{\mathbf{f}} & =\left[\tilde{f}_{1}, \tilde{f}_{2}, \ldots, \tilde{f}_{s}\right]
\end{aligned}
$$

where $\tilde{\mathbf{f}}$ indicates the reconstructed time series with length $s$. Note that one of the major problems in SSA is to find an appropriate group of eigentriples in order to reconstruct the desired component [29].

\subsection{Constrained $S S A$}

In order to extract the EEG periodic signals such as spindles, alpha, theta, and delta the following constrains can be applied:

I. Subspace Rejection; Each eigenvalue in the decomposition stage represents the variance of the signal in the direction of the corresponding PC. Eigenvalues related to the more powerful signals are located in the lower subspaces whereas less powerful signals occur in higher sub-225 spaces where the noise components usually arise. Therefore, the lower subspace is desirable here. To separate the lower subspace from the noise part, the following criterion should be applied to remove the noise part. All the PCs associated with the eigenvalues above $90 \%$ of the to-230 tal variance of the signal (i.e. the sum of all eigenvalues equals the total variance of the original time series) are omitted. Eigenvalue $\lambda_{j}$ is rejected if $j>\mathcal{L}$, where [19]:

$$
\mathcal{L}=\min \left\{h: \frac{\sum_{i=1}^{h} \lambda_{i}}{\sum_{i=1}^{l} \lambda_{i}}>0.9\right\}
$$

$h$ is defined as the number of eigenvalues whose overall energy is $90 \%$ of the total energy.

II. Periodic Component Extraction; a pseudo-periodic time series is factorised into some eigenvalue pairs using SSA $[30,28]$. Since the objective of this section is to extract the oscillatory components (i.e. sleep spindle, delta, theta, and alpha), the periodicity nature of these components is used here to choose the best subgroup of PCs for reconstruction of the brain waves. Thus, using the lower subspace indicated in the former subsection, only eigenvalues appeared as pairs are selected.

Following equation (7) and [17], the best subgroup of $\mathbf{X}$ is selected by minimisation of $\left\|\mathbf{X}-\mathbf{X}^{(d)}\right\|_{\mathcal{F}}$ where $\mathcal{F}$ stands for Frobenius norm and $\|\mathbf{X}\|_{\mathcal{F}}^{2}=\sum_{j=1}^{r} \lambda j$ and $\lambda j=\left\|\mathbf{X}_{j}\right\|_{\mathcal{F}}^{2}$ for $j=1, \cdots, r$. The ratio $\lambda j /\left\|\mathbf{X}_{j}\right\|^{2}$ is therefore the contribution of the trajectory matrix generated by the corresponding eigentriple $\left(\sqrt{\lambda_{j}}, \mathbf{q}_{j}, \mathbf{v}_{j}\right)$. However, the following points are important for the eigenvalue pairs selection $[19,28]$ :

- The possibility that the selected eigenvalue pairs belong to noise components.

- The possibility of having two equal eigenvalues is low mainly due to noise effect.

Therefore, in order to acquire the actual periodic pair, the eigenvalue pairs $\lambda_{j}$ and $\lambda_{i}$ are chosen as a pair only if all of the following circumstances are satisfied:

$i$. $i$ and $j$ are less than $\mathcal{L}$, where $\mathcal{L}$ is defined in (10) to discards all eigenvalues assumed to be associated with noise ii. $\lambda_{j}, \lambda_{j+1}|\quad| \lambda_{j+1}-\lambda_{j}|=\min | \lambda_{i}-\lambda_{i+1} \mid \quad \forall 1<i<l$ iii. $\left|1-\frac{\lambda_{j}}{\lambda_{i}}\right|<\mathcal{K}$

where the value of $\mathcal{K}$ can be changed according to the waveforms amplitude. Hence, a specific $\mathcal{K}$ value is set for each PC. Alpha, theta, and delta contain higher amplitudes than spindles. Therefore, according to what was discussed earlier, since their eigenvalues are within the lower subspaces, higher thresholds are chosen for them $(\mathcal{K}=0.05)$. On the other hand, for spindles with lower amplitudes, the eigenvalues are skewed towards right and thus a lower value for $\mathcal{K}$ is selected $(\mathcal{K}=0.005)$. If no eigenvalue pairs are obtained, it simply means that the component does not exist in the given time domain signal [28]. However, if some eigenvalues is chosen, the highest peak in the Fourier transform of the associated eigenvectors is relevant to the frequency of the periodic component [31]. Therefore, the power spectrum density is used to estimate the related frequency. The peak is required to fall into the desired frequency range (i.e. spindles $(12-16 \mathrm{~Hz})$, alpha $(8-12 \mathrm{~Hz})$, theta $(4-7 \mathrm{~Hz})$, and SWA $(1-4 \mathrm{~Hz}))$. 
K-complex has a different structure compared to spinand other mentioned sleep EEG waves. It abruptly appears in the EEG signal with larger amplitude in a shorter period of time. Subsequently, the extraction of $\mathrm{K}$-complex is relatively easy. The distribution of this waveform has a greater kurtosis than the rest of the EEG signal. of the signals reconstructed from a group of eigenvalues and their associated eigenvectors constitute the detection of K-complex.

Parameter Settings: The size of embedding window $l$ (num-

245 ber of columns of trajectory matrix) should make a compromise between low computational complexity and information quality. The embedding window should be sufficiently large to capture at least one period of the expected periodic signal. In addition, it has been suggested that

${ }_{250} l$ should not be more than $n / 2$ [17]. Window length is also selected based on the lowest frequency of interest $F w$ $\left(l>=\frac{F w}{F s}\right)[19]$. Therefore, by setting $l$ to 200 , more than two cycles of the oscillatory components are covered by the window (Fs=sampling frequency of $256 \mathrm{~Hz}$ ).

\subsection{Feature Extraction}

SSA is applied to the EEG signal for decomposing each 10 second segment into different frequency bands. Precise representation of the sleep EEG commonly demands ${ }_{30}$ for the localization of signal structure in time and frequency simultaneously. Therefore, appropriate temporal and spectral features are extracted from the EEG signals in different frequency ranges. These statistical descriptors are then used as the inputs to the classifier for classifica- ${ }_{305}$ tion purpose:

- Mean of absolute power in different frequency bands described as follows:

\begin{tabular}{cc}
\hline Mean Absolute Power & Frequency Bands \\
P1 & K-complex $(0.5-1.5 \mathrm{~Hz})$ \\
P2 & Delta $(1.5-4 \mathrm{~Hz})$ \\
P3 & Theta $(4-8 \mathrm{~Hz})$ \\
P4 & Alpha $(8-12 \mathrm{~Hz})$ \\
P5 & Sleep Spindle $(12-16 \mathrm{~Hz})$ \\
\hline
\end{tabular}

- Sum of power in all frequency bands: $P 6$

- Mean and standard deviation of ratios including $P 1$, P2, P3, P4, and P5 divided by $P 6$ produce 12 features.

- Mean and standard deviation of $\frac{P 1+P 2}{P 6}$ (delta activity),

- Mean and standard deviation of $\frac{P 4}{P 6}$ (alpha activity),

- Mean and standard deviation of $\frac{P 1+P 5}{P 6}$ (K-complex and sleep spindle), [15]
- Mean and standard deviation of ratios: $\frac{P 4}{P 2+P 3}, \frac{P 3}{P 2+P 4}$, $\frac{P 2}{P 3+P 4}[32]$.

\subsection{Multiclass SVM classification}

In a review of classification algorithms for EEG signals the performances of K-nearest neighbor (KNN), SVM, and linear discriminant analysis (LDA) have been compared [33]. Based on this study, they recommended an SVM classifier for this purpose. SVM has also been previously used for classification of sleep stages [34, 35].

The nature of SVMs are based on binary classification algorithms [17]. In case of linearly-separable data, SVM attempts to construct optimum hyperplane separating the training samples and the decision boundaries. Hyperplane construction whereby $v^{T} x+b=0$ ( $v$ represents the hyperplane coefficients vector and bias term is notated by $b$ ) in a way that the margin between the hyperplane and the nearest point is magnified to the maximum in a way which can fit into the quadratic-optimization problem [36].

In case of linearly-inseparable data, the support vec295 tors are taken into consideration and mapped to a high dimensional space in the hope that a segregating hyperplane can be detected. Kernel function is a nonlinear mapping function. Four common kernel functions include: linear, polynomial, radical basis function (RBF), and sigmoid. Finding the appropriate kernel differs for each problem. Nonetheless, the RBF kernel is extensively employed which forms the cornerstone of the current research as well.

Generally, the development of SVMs are for two-classes classification problems. However, the aim of this work is to automatic discrimination between four classes (awake, $\mathrm{N} 1+\mathrm{REM}, \mathrm{N} 2$, and SWS). For this objective, the design of multiclass SVM with a "one-against-all" approach is implemented.

\section{Results and Discussion}

In this paper, in order to to accentuate the usage of SSA as a preprocessing step for sleep events detection, MP time-frequency representation is employed. MP has been used to compare the effect of preprocessing data on the $\mathrm{T}-\mathrm{F}$ representation. The results prove that by using SSA as the method in the preprocessing stage, better representation and identification of the desired signal components can be achieved. The ultimate goal of the current work is to automatically classify the sleep stages. Therefore, the preprocessed data is used for feature extraction and then classification. This work concentrate on two stages; classifying different sleep stages and different sleep types. Hence, the following subsection show firstly the results of applying the proposed method on real data and T-F map of energy of these data. Next, the classification of sleep stages are provided and the results compared with those of raw data. Finally, the classification of sleep types is presented. 

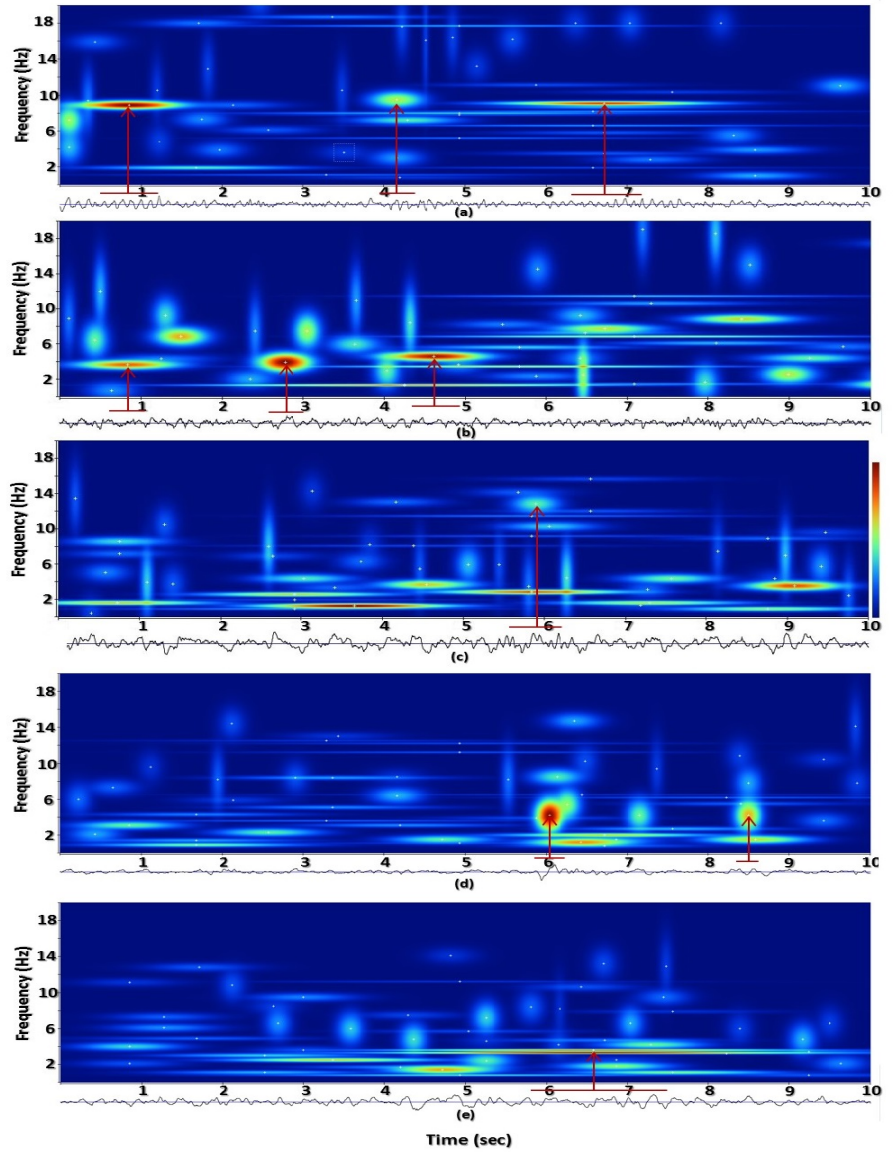

Figure 2: T-F representation of different sleep EEG stages for 10s segment; (a) awake, (b) N1, (c) N2, (d) N2, and (e) SWS

\subsection{Data}

Thirty-six healthy men and women each participated in two laboratory sessions, one involving a sleep extension protocol and the other a sleep restriction protocol. During each session PSG measures were recorded at a sampling rate of $256 \mathrm{~Hz}$ for an $\mathrm{SN}$ (8 hours), seven condition nights SE, (10 hours); SD, (6 hours) and a recovery night (12 hours) following a period of total sleep deprivation. This subset of dataset was recorded and validated in the Sleep Centre of the University of Surrey. The identification of sleep stage is performed by clinical experts using PSG signals including EOG, EMG, and EEG. Sleep is scored in

340 successive windows of 30 seconds according to the standard rules. For our analysis we have selected the data for ${ }_{360}$ different stages randomly.

\subsection{Real Data}

Using MP as explained in section 2.1, the sleep stages ${ }_{365}$ are detected and the sleep EEG structure is analysed using 10s segments of the single-channel EEG signal (C3-A2) by
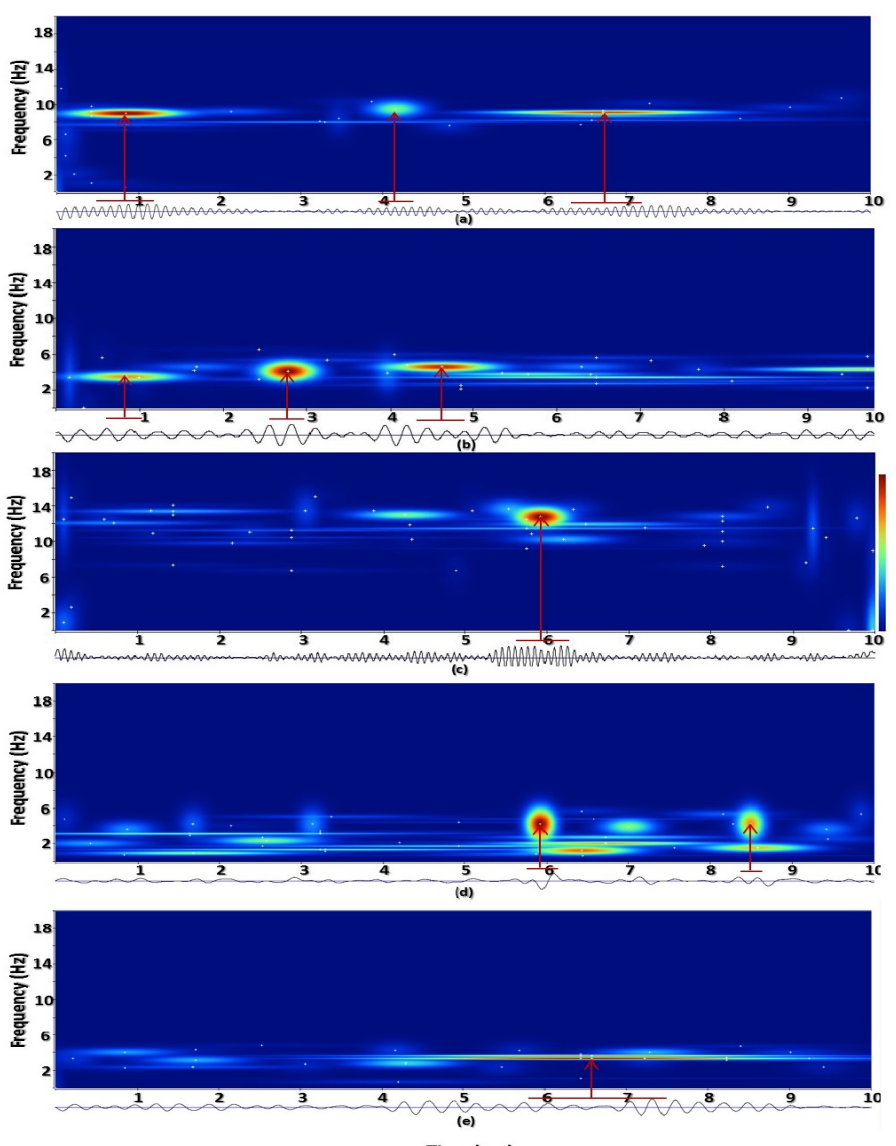

Time (sec)

Figure 3: T-F representation of different sleep EEG stages of the same subject as in Figure 2 using the proposed constrained SSA; (a) awake, marked alpha; (b) N1, marked theta; (c) N2, spindle; (d) N2 K-complex; and (e) SWS.

decomposing them as a weighted sum of basic waveforms $f_{\lambda}$. Figures 2 and 3 depict the T-F representation without and with preprocessing data respectively. Figure 2 represents 10 second EEG signals selected randomly from each stage including awake, stage 1, stage 2, and SWS. Accordingly, Figure 3 illustrates the extracted dominant features of each stage through SSA as follows: awake (alpha wave), stage 1 (theta wave), stage 2 (sleep spindle and k-complex), and SWS (delta wave). These specific sleep events in each stage are highlited by red points in each subfigure. Each blob displayed in the T-F map of energy is associated with one Gabor function.

As can be seen in Figure 3, using SSA and the constraint explained, all the brain waves and also spindles and $\mathrm{k}$ complexes are well separated. Sleep spindles are oscillatory components within the frequency range of $(12-14 \mathrm{~Hz})$ which last for $0.5-1$ second are visible as horizontal lines in the T-F domain and each spindle is described by only one atom which makes it possible to follow its evolution in time and space. The circular structure spread in fre- 

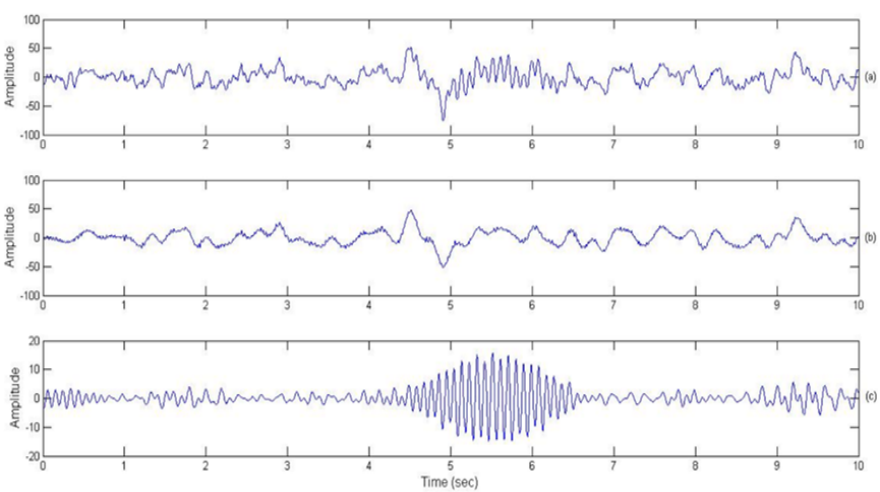

Figure 4: Separation of k-complex and sleep spindle from sleep EEG signal; (a) original signal, (b) the extracted k-complex, (c) the extracted sleep spindle

quency domain corresponds to k-complexes. In Figure 2, each Gabor function is fitted with both wanted and unwanted components. However, in Figure 3, only desired components are matched. Figures 2(c) and 3(c) share the occurrences of two spindles within the same 10s segment. Nevertheless, according to the chart scale, sleep spindle in Figure 3(c) has higher amplitude compared to those of 2(c). By plotting both original signal and the extracted signal using SSA, it is observed that the separated waves are located in exactly the same positions as their actual places in the original signals. It means that instead of visual analysis, it is possible to automatically localise the waveforms in time domain. Another significant usage of this method is that further to its separating characteristic ${ }_{410}$ it acts as a filter for preprocessing of the signals. Then, the T-F energy map clearly represents each wave by its specific frequency band.

\subsubsection{Feature Detection Experiment}

For this experiment an 8 second, $N=2048$ sample of the real data which shows the transition of two sleep features: sleep spindle and k-complex, is selected. Employing the previously discussed procedure for detection of these two features in Constrained SSA section, the sleep spindles and k-complexes can be well separated from EEG signal. ${ }_{420}$ Figure 4(a-c) illustrates how constrained SSA can be utilized to separate signal into different components simultaneously.

In order to better illustrate the performance of the algorithm, the T-F map of energy of the original signal and the extracted components are represented in Figure 5(a-c). $\quad{ }_{425}$

\subsection{Classification of Awake/N1+REM/N2/SWS}

The single-channel EEG signals from half of the data were utilized to train the classifier. The rest of the data were utilized to test the reconstructed model. Table 1 illus-430 trates the statistical description of the training and testing data by 30 second EEG epochs. Most of automatic sleep stage classification methods are employed by different PSG
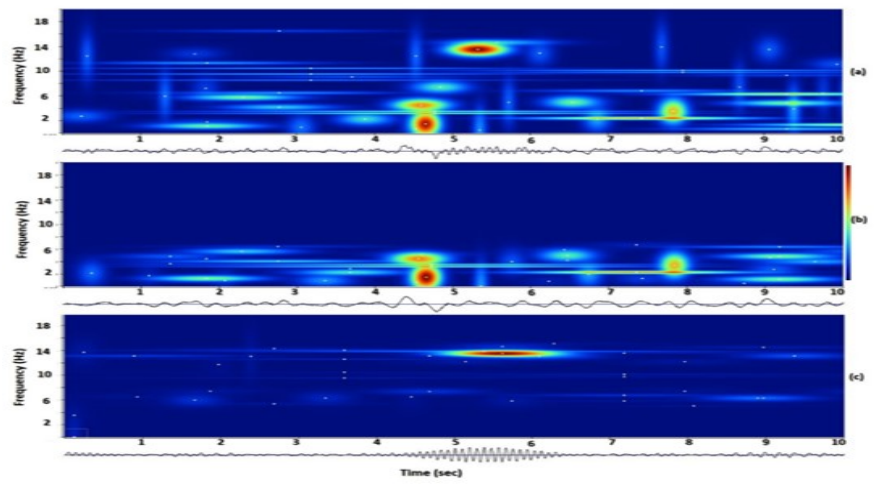

Figure 5: T-F representation of EEG signals of Figure 4; (a) original signal, (b) the extracted k-complex, (c) the extracted sleep spindle

Table 1: Information of the training and testing groups

\begin{tabular}{lll}
\hline & $\begin{array}{l}\text { Training } \\
(\text { Epochs })\end{array}$ & $\begin{array}{l}\text { Testing } \\
\text { (Epochs) }\end{array}$ \\
\hline \hline Awake & 196 & 200 \\
N1+REM & 723 & 804 \\
N2 & 912 & 1017 \\
N3 & 569 & 579 \\
Total Epochs & 2400 & 2600
\end{tabular}

single channel EEG signal.

Since N1 and REM have similar characteristics they can be merged into one class. Hence, we attempt to classify four sleep stages consisting of awake, N1 + REM, N2, and SWS. SVM utilized the training data to find the hyperplane which maximize the margin between the classes. Afterwards, the optimum classification is achieved through applying the separating hyperplane to the testing data.

In order to evaluate the classifier performance, accuracy, sensitivity, and specificity are calculated. The accuracy, sensitivity, and specificity are defined as follows:

Accuracy: $\frac{\text { number of correct decisions }}{\text { total number of cases }}$
Sensitivity: $\frac{\text { number of true positive decisions }}{\text { number of actually positive cases }}$
Specificity: $\frac{\text { number of true negative decisions }}{\text { number of actually negative cases }}$

These statistical comparisons are employed over SN data for both before and after preprocessing are shown in Table 2. From accuracy viewpoint, using SSA, the overall classification performance has been improved. There is in average $12.1 \%$ performance improvement in accuracy by applying the proposed method. The significant result achieved here is of immense value since it improves the automatic sleep stage classification to a large extent. When it comes to sensitivity, it is incremented by $14.5 \%$ in average for all the stages from before to after preprocessing.

Following the same strategy, four sleep stages are classified for SE and SD nights. The result for SE before and after SSA preprocessing are brought in Table 3. The same 
Table 2: SVM classification results for sleep normal (SN) data before and after applying SSA

\begin{tabular}{cccc|ccc}
\hline & \multicolumn{3}{c}{ Before Applying SSA } & \multicolumn{3}{c}{ After Applying SSA } \\
\cline { 2 - 4 } Awake and sleep stages & Accuracy\% & Sensitivity\% & Specificity\% & Accuracy\% & Sensitivity\% & Specificity\% \\
\hline Awake & $69.3 \pm 0.16$ & $53.7 \pm 0.09$ & $86.7 \pm 0.08$ & $81.2 \pm 0.14$ & $73.1 \pm 0.07$ \\
N1+REM & $62.0 \pm 0.12$ & $31.3 \pm 0.06$ & $85.1 \pm 0.06$ & $79.2 \pm 0.09$ & $48.2 \pm 0.10$ & $91.0 \pm 0.09$ \\
N2 & $77.6 \pm 0.13$ & $67.8 \pm 0.12$ & $86.2 \pm 0.07$ & $88.1 \pm 0.09$ & $78.9 \pm 0.06$ & $90.0 \pm 0.02$ \\
SWS & $77.2 \pm 0.03$ & $71.8 \pm 0.01$ & $89.4 \pm 0.01$ & $86.0 \pm 0.05$ & $82.5 \pm 0.12$ & $92.1 \pm 0.03$ \\
Total Stages & $71.5 \pm 0.11$ & $56.1 \pm 0.09$ & $86.8 \pm 0.04$ & $83.6 \pm 0.07$ & $70.6 \pm 0.14$ \\
\hline
\end{tabular}

Table 3: SVM classification results for sleep extension (SE) data before and after applying SSA

\begin{tabular}{cccc|ccc}
\hline \multirow{2}{*}{ Awake and sleep stages } & \multicolumn{3}{c}{ Before Applying SSA } & \multicolumn{3}{c}{ After Applying SSA } \\
\cline { 2 - 4 } \cline { 6 - 7 } & Accuracy\% & Sensitivity\% & Specificity\% & Accuracy\% & Sensitivity\% & Specificity\% \\
\hline Awake & $76.6 \pm 0.07$ & $74.2 \pm 0.05$ & $84.6 \pm 0.04$ & $83.8 \pm 0.07$ & $80.7 \pm 0.03$ \\
N1+REM & $59.3 \pm 0.12$ & $29.2 \pm 0.04$ & $81.3 \pm 0.03$ & $61.4 \pm 0.09$ & $40.1 \pm 0.10$ & $81.0 \pm 0.02$ \\
N2 & $81.2 \pm 0.06$ & $68.5 \pm 0.08$ & $81.8 \pm 0.04$ & $87.5 \pm 0.10$ & $73.1 \pm 0.07$ & $91.7 \pm 0.06$ \\
SWS & $74.9 \pm 0.04$ & $69.9 \pm 0.11$ & $84.7 \pm 0.03$ & $87.1 \pm 0.10$ & $79.5 \pm 0.01$ & $92.3 \pm 0.04$ \\
Total Stages & $73.0 \pm 0.10$ & $65.4 \pm 0.01$ & $83.1 \pm 0.07$ & $79.8 \pm 0.08$ & $68.3 \pm 0.11$ \\
\hline
\end{tabular}

Table 4: SVM classification results for sleep deprivation (SD) data before and after applying SSA

\begin{tabular}{|c|c|c|c|c|c|c|}
\hline \multirow[b]{2}{*}{ Awake and sleep stages } & \multicolumn{3}{|c|}{ Before Applying SSA } & \multicolumn{3}{|c|}{ After Applying SSA } \\
\hline & Accuracy $\%$ & Sensitivity\% & Specificity\% & Accuracy $\%$ & Sensitivity \% & Specificity \% \\
\hline Awake & $79.6 \pm 0.10$ & $63.9 \pm 0.01$ & $88.2 \pm 0.08$ & $83.6 \pm 0.12$ & $79.6 \pm 0.07$ & $98.0 \pm 0.05$ \\
\hline $\mathrm{N} 1+\mathrm{REM}$ & $61.4 \pm 0.09$ & $48.0 \pm 0.06$ & $83.9 \pm 0.03$ & $70.6 \pm 0.09$ & $52.8 \pm 0.10$ & $90.4 \pm 0.08$ \\
\hline $\mathrm{N} 2$ & $67.3 \pm 0.12$ & $66.8 \pm 0.08$ & $83.8 \pm 0.08$ & $77.5 \pm 0.03$ & $74.3 \pm 0.07$ & $94.4 \pm 0.08$ \\
\hline SWS & $78.1 \pm 0.14$ & $66.9 \pm 0.07$ & $88.1 \pm 0.03$ & $87.1 \pm 0.02$ & $81.7 \pm 0.13$ & $93.2 \pm 0.07$ \\
\hline Total Stages & $71.6 \pm 0.11$ & $61.4 \pm 0.09$ & $86.0 \pm 0.08$ & $79.7 \pm 0.01$ & $72.1 \pm 0.07$ & $94.0 \pm 0.05$ \\
\hline
\end{tabular}
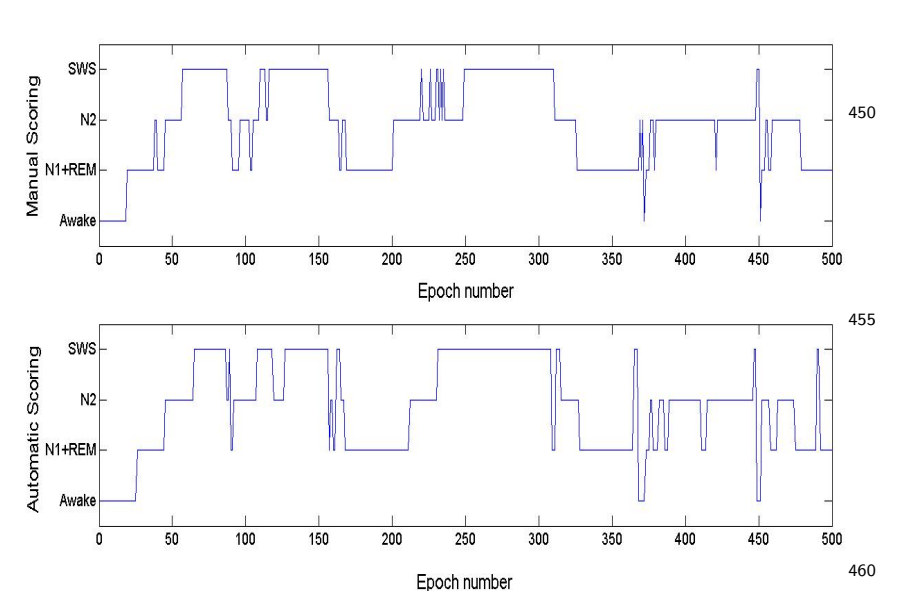

Figure 6: Representative four sleep stage classification of 500 epochs acquired using clinical expert (top) and SSA (bottom).

results for $\mathrm{SD}$ can be seen in Table 4 . Regarding SE, the ${ }_{465}$ accuracy for total stages hits the peak of $79.8 \pm 0.08 \%$ after preprocessing compared with $73.0 \pm 0.10 \%$ before that. Similar result can be seen regarding SD with performance improvement in sensitivity from $61.4 \pm 0.09 \%$ to $72.1 \pm 0.07 \%$ using the proposed method. In summary,470 The accuracy of all sleep stages for 3 types of sleep shows a significant improvement except stage N1+REM, see tables $2-4$. We consider S1 and REM as a single stage due to their similarity in EEG pattern. Despite that, the detection of N1+REM has not significant changes amongst ${ }_{475}$ sleep stages. Since N1 is a transition stage between wake- fulness and asleep, it is still a challenging issue to find an appropriate feature that would separate N1 from N2 and wakefulness.

The k-fold cross validation ( $\mathrm{CV}$ ) technique is used to validate the classifier. The classifier performance shows $11.09 \%$ error though 10-fold CV method. As another example to shows the efficiency of our proposed method, Figure 6 represents both clinical expert and automatic sleep stage classification for 500 epochs 30 seconds long.

\subsection{Classification of $S E / S N / S D$}

For all the sleep types, it is desirable to compute different spindle characteristics such as spindle mean amplitude, density, frequency, and lastly the duration. Figure 7 depicts the mean and the standard error of the mean (SEM) for all subjects. The substantial point here is that SN's values for the duration, amplitude, density, and frequency always fall into a domain limited to the SE nights on one hand as well as the SD nights on the other for all four cases. The mean spindle amplitude and duration are 17.20 (s.d. $=1.1$ ) and 1.25 (s.d. =0.08) during SN which increase to 18.90 (s.d. $=0.99$ ) and 1.32 (s.d. $=0.08$ ) during $\mathrm{SD}$ respectively. Despite this increase, SE's mean amplitude and duration values significantly reduce to 15.07 (s.d. = 0.89 ) as well as 0.94 (s.d. = 0.09) compared to SN. With regard to the density as well as the frequency, SE's mean values increase to 1.34 (s.d. $=0.09)$ and $13.59($ s.d. $=0.2)$ respectively. $\mathrm{SD}$ nights however, diminish to 1.05 (s.d. = 0.1 ) and 13.41 (s.d. $=0.1$ ) for each aforementioned parameters in contrast to SN nights. Considering the impact of 
SE on the characteristics of spindles is novel to our best knowledge so far.

These results not only confirm but also further expand that the characteristics of sleep spindle are remarkably influenced by homeostatic sleep pressure. Our findings in terms of SD validate the reduction of spindle density after SD $[24,25]$. Previous works believed that the only significant change occurs with the density $[24,37]$. While, our results prove that the duration, amplitude and frequency also change noticeably for SD nights compared to SN nights. Similar to ours, the work in [25] confirms that all the values change for SD and state that the changes are negligible for the duration. Our work, however, steps further by proving that a significant difference is also exist for duration. This stems from the fact that what is detected with our approach for the automatic detection of the sleep spindles is not subject to any change of scale.

The growth observed in the spindle amplitude of SD case as well as the reduction seen in the frequency advocate the hypothesis of a greater level of synchronization in TC when homeostatic sleep pressure is enhanced [25]. Furthermore our findings further stress that with SE, the spindle amplitude goes down whereas the frequency rises. In view of this result, we argue that it is likely that there is a lower level of synchronisation in TC when homeostatic sleep pressure is low.

At the next stage, the previously mentioned values calculated for different spindle parameters act as the input features for the SVM classifier to categorise sleep types such as SE, SN, and SD. The associated accuracy, sensitivity and specificity are shown in Table 5.

\section{Conclusions}

In this article we incorporated the MP-based T-F representation of sleep EEG together with an effective approach for refining the data. The refining procedure is ${ }^{53}$ based on a known method namely SSA. SSA not only provides all the necessary features of the data for the classification of sleep stages, but also removes the undesired components to considerably improve the classification performance. In addition, thanks to the SSA, parameterising the sleep spindles of SN, SD, and SE has noticeably enhanced 540 to an extent that the sleep types were classified. The proposed constrained SSA decomposes the signals into their constituent components in a supervised manner in order to ensure that the desired components are well preserved ${ }^{545}$ while the undesired ones are set aside. The proposed hybrid method paves the way for further analysis of sleep EEG to enable characterisation of sleep abnormalities and many mental and physical disorders. This work has the ${ }^{550}$ potential for more diverse set of subjects and features for classifying other set of sleep stages. In addition, here, only normal subjects are involved. Further studies will include those with sleep disorders and other related abnormalities. ${ }^{555}$

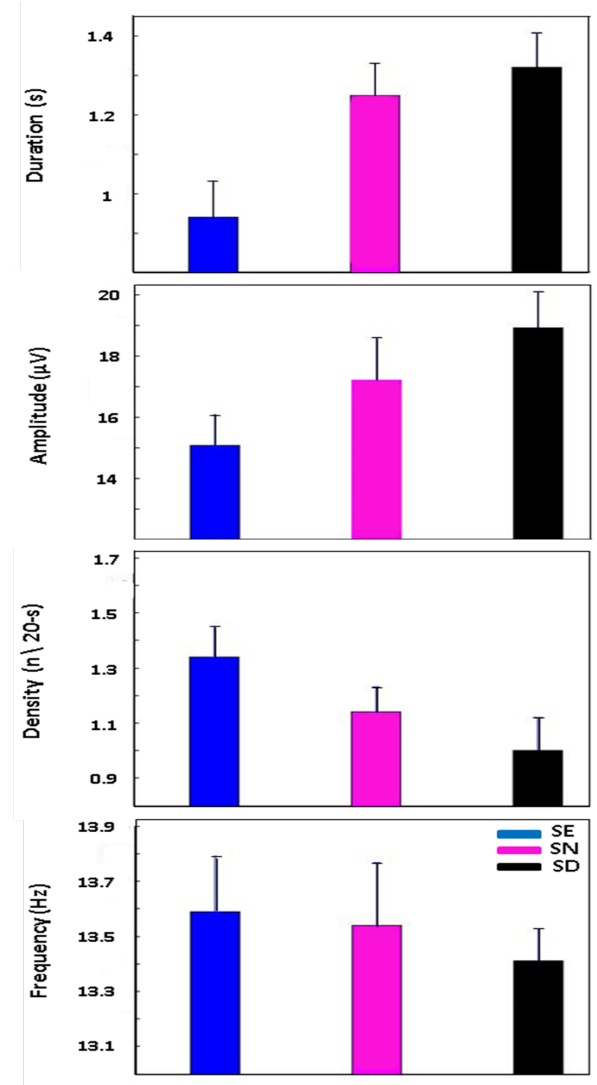

Figure 7: Mean duration, amplitude, density (number of sleep spindles per 20-s epoch), and frequency of sleep spindles during SE, SN, and SD. Error bars shows the standard error of mean (mean SEM)

\section{ACKNOWLEDGMENT}

The authors wish to thank Prof. Derk-Jan Dijk and Dr. Emma Arbon from the Surrey Sleep Research Centre, University of Surrey, Guildford, UK, for providing the sleep EEG data.

\section{References}

[1] K. Susmakova, Human sleep and sleep EEG, Journal of Institute of Measurements Science SAS, Slovak Academy of Sciences 4 (2) (2004) 59-74.

[2] J. M. Kortelainen, M. O. Mendez, A. M. Bianchi, M. Matteucci, S. Cerutti, Sleep staging based on signals acquired through bed sensor, IEEE Transactions on Information Technology in Biomedicine 14 (3) (2010) 776-785.

[3] A. Rechtschaffen, A. Kales, A manual of standardized terminology, techniques and scoring system for sleep stages of human subjects, US Department of Health, Education and Welfare, Public Health Service, National Institutes of Health, National Institute of Neurological Diseases and Blindness, Neurological Information Network, 1968.

[4] C. Iber, The AASM manual for the scoring of sleep and associated events: rules, terminology and technical specifications, American Academy of Sleep Medicine, 2007.

[5] J. W. Shephard, Atlas of sleep medicine, Futura Publishing Compony, 1991.

[6] S. Sanei, Adaptive processing of brain signals, John Wiley \& Sons, 2013. 
Table 5: SVM classification results for SE, SN, and SD via SSA.

\begin{tabular}{|c|c|c|c|c|c|c|}
\hline \multirow[b]{2}{*}{ Type of Sleep } & \multicolumn{3}{|c|}{ Before Applying SSA } & \multicolumn{3}{|c|}{ After Applying SSA } \\
\hline & Accuracy $\%$ & Sensitivity\% & Specificity\% & Accuracy $\%$ & Sensitivity \% & Specificity \% \\
\hline Extension & $72.9 \%$ & $72.3 \%$ & $88.2 \%$ & $88.3 \%$ & $84.0 \%$ & $95.6 \%$ \\
\hline Normal & $65.6 \%$ & $56.9 \%$ & $81.3 \%$ & $78.9 \%$ & $73.4 \%$ & $89.3 \%$ \\
\hline Deprivation & $71.7 \%$ & $66.9 \%$ & $79.9 \%$ & $84.3 \%$ & $79.9 \%$ & $90.2 \%$ \\
\hline
\end{tabular}

[7] M. K. C. Hublin, M. Partinen, J. Kaprio, Sleep and mortality: a population-based 22-year follow-up study, Sleep 30 (10) (2007) 1245.

[8] N. A. Collop, Scoring variability between polysomnography technologists in different sleep laboratories, Sleep medicine 3 (1) (2002) 43-47.

[9] S. Enshaeifar, S. Kouchaki, C. C. Took, S. Sanei, Quaternion singular spectrum analysis of electroencephalogram with625 application in sleep analysis, IEEE Transactions on Neural Systems and Rehabilitation Engineering 24 (1) (2016) 57-67. doi:10.1109/TNSRE.2015.2465177.

[10] J. Virkkala, J. Hasan, A. Värri, S. Himanen, K. Müller, Automatic sleep stage classification using two-channel electro-630 oculography, Journal of neuroscience methods 166 (1) (2007) 109-115.

[11] C. Berthomier, X. Drouot, M. Herman-Stö̈ca, P. Berthomier, J. Prado, D. Bokar-Thire, O. Benoit, J. Mattout, M. d'Ortho, Automatic analysis of single-channel sleep EEG: validation in635 healthy individuals, Sleep 30 (11) (2007) 1587.

[12] L. Fraiwan, K. Lweesy, N. Khasawneh, H. Wenz, H. Dickhaus, Automated sleep stage identification system based on timefrequency analysis of a single EEG channel and random forest classifier, Computer methods and programs in biomedicine 640 108 (1) (2012) 10-19.

[13] J. Żygierewicz, K. J. Blinowska, P. J. Durka, W. S. S. Niemcewicz, W. Androsiuk, High resolution study of sleep spindles, Clinical Neurophysiology 110 (12) (1999) 2136-2147.

[14] M. S. Kim, Y. C. Cho, B. Abibullaev, H. D. Seo, Analysis 645 of brain function and classification of sleep stage EEG using daubechies wavelet, Sens. Mater 20 (1) (2008) 1-15.

[15] E. Oropesa, H. L. Cycon, M. Jobert, Sleep stage classification using wavelet transform and neural network, International Computer Science Institute, 1999.

[16] U. Malinowska, P. J. Durka, K. J. Blinowska, W. Szelenberger, A. Wakarow, Micro- and macrostructure of sleep EEG, IEEE Engineering in Medicine and Biology Magazine 25 (4) (2006) $26-31$.

[17] N. Golyandina, V. Nekrutkin, A. Zhigljavsky, Analysis of time655 series structure: SSA and related techniques, CRC Press, 2010.

[18] S. Sanei, H. Hassani, Singular spectrum analysis of biomedical signals, CRC Press, 2015.

[19] F. Ghaderi, H. R. Mohseni, S. Sanei, Localizing heart sounds in respiratory signals using singular spectrum analysis, IEEE660 Transactions on Biomedical Engineering 58 (12) (2011) 33603367.

[20] S. Sanei, T. Lee, V. Abolghasemi, A new adaptive line enhancer based on singular spectrum analysis, IEEE Transactions on Biomedical Engineering 59 (2) (2012) 428-434.

[21] D. Jarchi, C. Wong, T. B. a. A. G. R. Kwasnicki, H. Mark and G. Yang, Gait parameter estimation from a miniaturized earworn sensor using singular spectrum analysis and longest common subsequence, IEEE Transactions on Biomedical Engineering 61 (4) (2014) 1261-1273.

[22] S. Kouchaki, S. Sanei, E. Arbon, D.-J. Dijk, Tensor based singular spectrum analysis for automatic scoring of sleep eeg, IEEE Transactions on Neural Systems and Rehabilitation Engineering, 23 (1) (2015) 1-9.

[23] N. Ward, S. M. Cowie, Rosen, V. Roldao, M. D. Villa, T. McDonagh, A. Simonds, M. Morrell, Utility of overnight pulse oximetry and heart rate variability analysis to screen for sleepdisordered breathing in chronic heart failure, Thorax 67 (11)
(2012) 1000-1005.

[24] D.-J. Dijk, B. Hayes, C. A. Czeisler, Dynamics of electroencephalographic sleep spindles and slow wave activity in men: effect of sleep deprivation, Brain Research 626 (12) (1993) 190 $-199$.

[25] V. Knoblauch, W. L. J. Martens, A. Wirz-Justice, C. Cajochen, Human sleep spindle characteristics after sleep deprivation, Clinical Neurophysiology 114 (12) (2003) 2258 -67.

[26] S. G. Mallat, Z. Zhang, Matching pursuits with time-frequency dictionaries, IEEE Transactions on Signal Processing 41 (12) (1993) 3397-15

27] S. Sanei, M. Ghodsi, H. Hassani, An adaptive singular spectrum analysis approach to murmur detection from heart sounds, Elsevier Journal of Medical Engineering and Physics 33 (3) (2011) $362-367$.

[28] J. Mamou, E. J. Feleppa, Singular spectrum analysis applied to ultrasonic detection and imaging of brachytherapy seeds, Journal of the Acoustical Society of America 121 (3) (2007) 17901801.

[29] S. Enshaeifar, S. Sanei, C. C. Took, An eigen-based approach for complex-valued forecasting, in: Acoustics, Speech and Signal Processing (ICASSP), 2014 IEEE International Conference on, IEEE, 2014, pp. 6014-6018.

[30] R. Vautard, P. Yiou, M. Ghil, Singular-spectrum analysis: A toolkit for short, noisy chaotic signals, Elsevier Journal of Physica D: Nonlinear Phenomena 58 (1) (1992) 95-126.

[31] Y. Tao, E. C. M. Lam, Y. Y. Tang, Feature extraction using wavelet and fractal, Elsevier Journal of Pattern Recognition Letters 22 (3) (2001) 271-287.

[32] F. Ebrahimi, a. E. E. M. Mikaeili, H. Nazeran, Automatic sleep stage classification based on EEG signals by using neural networks and wavelet packet coefficients, in: Engineering in Medicine and Biology Society, 2008. EMBS 2008. 30th Annual International Conference of the IEEE, 2008, pp. 1151-1154.

[33] F. Lotte, M. Congedo, A. Lcuyer, F. Lamarche, B. Arnaldi, A review of classification algorithms for EEG-based braincomputer interfaces, Journal of neural engineering 4 (2) (2007) R1

[34] G. Zhu, Y. Li, P. P. Wen, in: F. Zanzotto, S. Tsumoto, N. Taatgen, Y. Yao (Eds.), Brain Informatics, Vol. 7670 of Lecture Notes in Computer Science, 2012.

[35] M. Adnane, Z. Jiang, Z. Yan, Sleep-wake stages classification and sleep efficiency estimation using single-lead electrocardiogram, Elsevier Journal of Expert Systems with Applications 39 (1) (2012) 1401-13.

[36] V. Vapnik, Statistical learning theory, Vol. 1, Wile, NY, 1998.

[37] L. D. Gennaro, M. Ferrara, M. Bertini, Effect of slow-wave sleep deprivation on topographical distribution of spindles, Behavioural Brain Research 116 (1) (2000) 55 - 59 